\title{
Charged fermion tunneling from electrically and magnetically charged rotating black hole in de Sitter space
}

\author{
M. M. Stetsko ${ }^{\mathrm{a}}$ \\ Department of Theoretical Physics, Ivan Franko National University of Lviv, 12 Drahomanov Str., Lviv 79005, Ukraine
}

Received: 21 July 2013 / Accepted: 29 November 2013 / Published online: 23 January 2014

(c) The Author(s) 2014. This article is published with open access at Springerlink.com

\begin{abstract}
Thermal radiation of electrically charged fermions from a rotating black hole with electric and magnetic charges in de Sitter space is considered. The tunneling probabilities for outgoing and incoming particles are obtained and the Hawking temperature is calculated. The relation for the classical action for the particles in the black hole's background is also found.
\end{abstract}

\section{Introduction}

Hawking radiation has attracted a lot of attention since it was proposed [1]. Different methods have been applied [2] to its study. The semi-classical tunneling approach that was proposed by Kraus and Wilczek [3,4] has gained considerable interest recently. It was shown that the Hawking temperature is defined by the imaginary part of the emitted particle's action for the classically forbidden region near the horizon. To calculate it two methods were proposed. The first one is the so-called null-geodesic method proposed by Parikh and Wilczek [5] which is based on the fact that the imaginary part of the action is caused by the integration of the radial momentum $p_{\mathrm{r}}$ for the emitted particles. The second method is based on the relativistic Hamilton-Jacobi equation, and the imaginary part of the action can be obtained after integration of that equation [6]. This second approach can be treated as an extension of the complex path method proposed by Padmanabhan et al. [7-9].

The tunneling approach based on the Hamilton-Jacobi equation at first was applied to the emission of scalar particles. Then it was successfully applied to the vast area of well-known and exotic spacetimes, in particular the Kerr and Kerr-Newman ones [10,11], the Taub-NUT spacetime [12] the Gödel spacetime [13], BTZ black holes [14], and dynamical black holes [15]. A review of the tunneling method is considered in Ref. [16], where further references can be found.

a e-mail:mstetsko@gmail.com; mykola@ktf.franko.lviv.ua
The tunneling approach was also successfully applied to the tunneling of fermions. In their seminal work, Kerner and Mann used the Dirac equation instead of the HamiltonJacobi one to obtain the temperature of the emitted fermions and showed that for a chosen type of spacetime the temperature of the emitted fermions would be the same as the temperature of scalar particles [17]. That method was later applied to different kinds of black hole spacetimes, including the Reissner-Nordström one [18], the Kerr-Newman one $[19,20]$, dilatonic black holes [21], BTZ black holes [22], black holes in Hořava-Lifshitz gravity [23,24], accelerating and rotating black holes $[25,26]$, and rotating black strings [27].

In our work, we consider the Kerr-Newman-de Sitter black hole which carries both electric and magnetic charges. Using the Kerner-Mann procedure, we consider the emission of charged spin $1 / 2$ particles. We show that in the presence of electric and magnetic charges of the black hole the variables of the Dirac equation can also be separated and as a consequence the temperature can be found. We also find the quasiclassical action of an emitted particle.

\section{Charged spin 1/2 particle tunneling from Kerr-Newman-de Sitter black hole}

Emission of charged particles with spin $1 / 2$ was considered independently in Ref. [19,20]. We consider emission of a charged spin $1 / 2$ particle from a Kerr-Newman-de Sitter black hole which carries both electric and magnetic charges (dyonic black hole). The Kerr-Newman-de Sitter black hole's metric in Boyer-Lindquist coordinates takes the form

$$
\begin{aligned}
\mathrm{d} s^{2}= & -\frac{\Delta}{\rho^{2}}\left(\mathrm{~d} t-\frac{a \sin ^{2} \theta}{\Xi} \mathrm{d} \varphi\right)^{2}+\frac{\rho^{2}}{\Delta} \mathrm{d} r^{2}+\frac{\rho^{2}}{\Delta_{\theta}} \mathrm{d} \theta^{2} \\
& +\frac{\Delta_{\theta} \sin ^{2} \theta}{\rho^{2}}\left(\frac{R^{2}}{\Xi} \mathrm{d} \varphi-a \mathrm{~d} t\right)^{2},
\end{aligned}
$$


where $R^{2}=r^{2}+a^{2}, \rho^{2}=r^{2}+a^{2} \cos ^{2} \theta, \Delta=R^{2}(1-$ $\left.r^{2} / l^{2}\right)+Q_{\mathrm{e}}^{2}+Q_{\mathrm{m}}^{2}-2 M r, \Xi=1+a^{2} / l^{2}, \Delta_{\theta}=$ $1+a^{2} / l^{2} \cos ^{2} \theta$. Here $a=J / M$ is the angular momentum parameter and the other parameter, $l^{2}=3 / \Lambda$, is defined by the cosmological constant; the parameters $Q_{\mathrm{e}}$ and $Q_{\mathrm{m}}$ are electric and magnetic charges, respectively. It is known that the black hole's horizons can be found from the equation $\Delta=0$, and in the case of Kerr-Newman-de Sitter metrics we have four roots. The largest one is the cosmological horizon $r_{\mathrm{c}}(\mathrm{CH})$, the minimal positive root is the Cauchy horizon $r_{i}$, and the intermediate root is the event horizon $r_{+}$. The last root is negative and is not taken into consideration.

The components of the electromagnetic potential take the form

$$
\begin{aligned}
& A_{t}=-\frac{1}{\rho^{2}}\left(Q_{\mathrm{e}} r-Q_{\mathrm{m}} a \cos \theta\right), \\
& A_{\varphi}=\frac{1}{\Xi}\left(Q_{\mathrm{e}} \frac{r a}{\rho^{2}} \sin ^{2} \theta+Q_{\mathrm{m}}\left( \pm 1-\frac{R^{2}}{\rho^{2}} \cos \theta\right)\right) .
\end{aligned}
$$

We note that the magnetic part of the vector potential contains a string singularity. The two signs in that term correspond to two choices of gauge. Taking into account the boundary conditions (matching of the potential at the boundary) allows one to avoid the divergence of the vector potential [28].

The Dirac equation for electrically charged particle takes the form

$i \gamma^{\mu}\left(D_{\mu}-\frac{i q}{\hbar} A_{\mu}\right) \psi+\frac{m}{\hbar} \psi=0$

where $D_{\mu}=\partial_{\mu}+\Omega_{\mu}, \Omega_{\mu}=\frac{1}{8} \Gamma_{\mu}^{\alpha \beta}\left[\gamma^{\beta}, \gamma^{\alpha}\right]$ and $\gamma^{\mu}$ matrices are obeyed to commutation relation:

$\left\{\gamma^{\mu}, \gamma^{\nu}\right\}=2 g^{\mu \nu} \hat{1}$

The representation for the $\gamma^{\mu}$ can be taken as follows:

$$
\begin{aligned}
& \gamma^{t}=\frac{\sqrt{K(r, \theta)}}{\rho \sqrt{\Delta \Delta_{\theta}}} \gamma^{0}, \quad \gamma^{r}=\frac{\sqrt{\Delta}}{\rho} \gamma^{3}, \quad \gamma^{\theta}=\frac{\sqrt{\Delta_{\theta}}}{\rho} \gamma^{1}, \\
& \gamma^{\varphi}=\frac{\Xi}{\sqrt{K(r, \theta)}}\left(\frac{\rho}{\sin \theta} \gamma^{2}-\frac{a\left(\Delta-R^{2} \Delta_{\theta}\right)}{\rho \sqrt{\Delta \Delta_{\theta}}} \gamma^{0}\right),
\end{aligned}
$$

where we denoted $K(r, \theta)=R^{4} \Delta_{\theta}-\Delta a^{2} \sin ^{2} \theta$, and the matrices $\gamma^{a}$ take the form

$$
\begin{array}{ll}
\gamma^{0}=\left(\begin{array}{cc}
0 & I \\
-I & 0
\end{array}\right) & \gamma^{1}=\left(\begin{array}{cc}
0 & \sigma_{1} \\
\sigma_{1} & 0
\end{array}\right) \\
\gamma^{2}=\left(\begin{array}{cc}
0 & \sigma_{2} \\
\sigma_{2} & 0
\end{array}\right) & \gamma^{3}=\left(\begin{array}{cc}
0 & \sigma_{3} \\
\sigma_{3} & 0
\end{array}\right)
\end{array}
$$

and $\sigma_{i}$ are the Pauli matrices. We note that a representation similar to (6) was used in [20].
We choose the wave functions with spin-up and spin-down in the form

$$
\begin{aligned}
& \psi_{\uparrow}=\left(\begin{array}{c}
A(t, r, \theta, \varphi) \\
0 \\
B(t, r, \theta, \varphi) \\
0
\end{array}\right) \exp \left(\frac{i}{\hbar} I_{\uparrow}(t, r, \theta, \varphi)\right) \\
& \psi_{\downarrow}=\left(\begin{array}{c}
0 \\
C(t, r, \theta, \varphi) \\
0 \\
B(t, r, \theta, \varphi)
\end{array}\right) \exp \left(\frac{i}{\hbar} I_{\downarrow}(t, r, \theta, \varphi)\right),
\end{aligned}
$$

where $I_{\uparrow}$ and $I_{\downarrow}$ are the action for the Dirac particles with spin-up $(\uparrow)$ and spin-down $(\downarrow)$ tunneling through the horizons.

Then we substitute (10) into the Dirac equation (4) and perform the quasiclassical approximation to obtain

$$
\begin{gathered}
B\left[-\frac{\sqrt{K(r, \theta)}}{\rho \sqrt{\Delta \Delta_{\theta}}} \partial_{t} I_{\uparrow}-\frac{\sqrt{\Delta}}{\rho} \partial_{r} I_{\uparrow}+\frac{\Xi a\left(\Delta-R^{2} \Delta_{\theta}\right)}{\rho \sqrt{\Delta \Delta_{\theta} K(r, \theta)}} \partial_{\varphi} I_{\uparrow}\right. \\
-\frac{q \sqrt{K(r, \theta)}}{\rho^{3} \sqrt{\Delta_{\theta}}}\left(Q_{\mathrm{e}} r-Q_{\mathrm{m}} a \cos \theta\right)-\frac{q a\left(\Delta-R^{2} \Delta_{\theta}\right)}{\rho \sqrt{\Delta \Delta_{\theta} K(r, \theta)}} \\
\left.\quad \times\left(Q_{\mathrm{e}} \frac{r a}{\rho^{2}} \sin ^{2} \theta+Q_{\mathrm{m}}\left( \pm 1-\frac{R^{2}}{\rho^{2}} \cos \theta\right)\right)\right]+m A=0 ;
\end{gathered}
$$

$$
\begin{aligned}
B[ & -\frac{\sqrt{\Delta_{\theta}}}{\rho} \partial_{\theta} I_{\uparrow}-\frac{i \Xi \rho}{\sqrt{K(r, \theta)} \sin \theta} \partial_{\varphi} I_{\uparrow}+\frac{i q \rho}{\sqrt{K(r, \theta)} \sin \theta} \\
& \left.\times\left(Q_{\mathrm{e}} \frac{r a}{\rho^{2}} \sin ^{2} \theta+Q_{\mathrm{m}}\left( \pm 1-\frac{R^{2}}{\rho^{2}} \cos \theta\right)\right)\right]=0 ;(12)
\end{aligned}
$$

$$
\begin{aligned}
A[ & \frac{\sqrt{K(r, \theta)}}{\rho \sqrt{\Delta \Delta_{\theta}}} \partial_{t} I_{\uparrow}-\frac{\sqrt{\Delta}}{\rho} \partial_{r} I_{\uparrow}-\frac{\Xi a\left(\Delta-R^{2} \Delta_{\theta}\right)}{\rho \sqrt{\Delta \Delta_{\theta} K(r, \theta)}} \partial_{\varphi} I_{\uparrow} \\
& +\frac{q \sqrt{K(r, \theta)}}{\rho^{3} \sqrt{\Delta \Delta_{\theta}}}\left(Q_{\mathrm{e}} r-Q_{\mathrm{m}} a \cos \theta\right)+\frac{q a\left(\Delta-R^{2} \Delta_{\theta}\right)}{\rho \sqrt{\Delta \Delta_{\theta} K(r, \theta)}} \\
& \left.\times\left(Q_{\mathrm{e}} \frac{r a}{\rho^{2}} \sin ^{2} \theta+Q_{\mathrm{m}}\left( \pm 1-\frac{R^{2}}{\rho^{2}} \cos \theta\right)\right)\right]+m B=0 ;
\end{aligned}
$$

$$
\begin{aligned}
B[ & -\frac{\sqrt{\Delta_{\theta}}}{\rho} \partial_{\theta} I_{\uparrow}-\frac{i \Xi \rho}{\sqrt{K(r, \theta)} \sin \theta} \partial_{\varphi} I_{\uparrow}+\frac{i q \rho}{\sqrt{K(r, \theta)} \sin \theta} \\
& \left.\times\left(Q_{\mathrm{e}} \frac{r a}{\rho^{2}} \sin ^{2} \theta+Q_{\mathrm{m}}\left( \pm 1-\frac{R^{2}}{\rho^{2}} \cos \theta\right)\right)\right]=0 .
\end{aligned}
$$

Note that in the first-order WKB approximation the terms proportional to $\Omega_{\mu}$ are omitted.

Suppose that the action $I_{\uparrow}$ takes the form

$$
I_{\uparrow}=-E t+J \varphi+W(r, \theta) .
$$


Now we substitute (15) into (11)-(14). In order to make these equations simpler, they are decomposed into the series near the horizon surface $[19,20]$ (here the decomposition in the vicinity of the event horizon is represented):

$$
\begin{aligned}
& B\left[\frac{R_{+}^{2} E-\Xi a J-q\left(Q_{\mathrm{e}} r_{+} \mp Q_{\mathrm{m}} a\right)}{\rho_{+} \sqrt{\Delta_{r}\left(r_{+}\right)\left(r-r_{+}\right)}}\right. \\
& \left.\quad-\frac{\sqrt{\Delta_{r}\left(r_{+}\right)\left(r-r_{+}\right)}}{\rho_{+}} W_{r}(r, \theta)\right]+m A=0 ; \\
& B\left[-\frac{\sqrt{\Delta_{\theta}}}{\rho_{+}} W_{\theta}(r, \theta)-\frac{i \rho_{+}}{R_{+}^{2} \sqrt{\Delta_{\theta}} \sin \theta}\right. \\
& \left.\quad \times\left(\Xi J-q\left[Q_{\mathrm{e}} \frac{r_{+} a}{\rho_{+}^{2}} \sin ^{2} \theta+Q_{\mathrm{m}}\left( \pm 1-\frac{R_{+}^{2}}{\rho_{+}^{2}} \cos \theta\right)\right]\right)\right]=0,
\end{aligned}
$$

$$
\begin{aligned}
& A\left[-\frac{R_{+}^{2} E-\Xi a J-q\left(Q_{\mathrm{e}} r_{+} \mp Q_{\mathrm{m}} a\right)}{\rho_{+} \sqrt{\Delta_{r}\left(r_{+}\right)\left(r-r_{+}\right)}}\right. \\
& \left.\quad-\frac{\sqrt{\Delta_{r}\left(r_{+}\right)\left(r-r_{+}\right)}}{\rho_{+}} W_{r}(r, \theta)\right]+m B=0 ; \\
& A\left[-\frac{\sqrt{\Delta_{\theta}}}{\rho_{+}} W_{\theta}(r, \theta)-\frac{i \rho_{+}}{R_{+}^{2} \sqrt{\Delta_{\theta}} \sin \theta}\right. \\
& \left.\quad \times\left(\Xi J-q\left[Q_{\mathrm{e}} \frac{r_{+} a}{\rho_{+}^{2}} \sin ^{2} \theta+Q_{\mathrm{m}}\left( \pm 1-\frac{R_{+}^{2}}{\rho_{+}^{2}} \cos \theta\right)\right]\right)\right]=0 .
\end{aligned}
$$

Here $\Delta_{r}\left(r_{+}\right)=2\left(r_{+}\left(1-a^{2} / l^{2}\right)-2 r_{+}^{3} / l^{2}-M\right), R_{+}^{2}=$ $r_{+}^{2}+a^{2}$ and $\rho_{+}^{2}=r_{+}^{2}+a^{2} \cos ^{2} \theta$.

It should be stressed that the coordinate $\theta$ in our decompositions in the vicinity of the horizon point $r_{+}$is not fixed similarly as in Ref. [20]. We also remark that in order to get the black hole's temperature in [12], the polar angle $\theta$ was fixed in the near-horizon metric. This was done for the following reason: if the coordinate $\theta$ is fixed the equations of motion can easily be integrated, especially in the null-geodesic approach. It was noted that the resulting temperature would not depend on the chosen angle $\theta$, so it can take an arbitrary value [12]. A similar situation was considered in Ref. [10], but there, to simplify the equations for the null geodesics, an additional transformation of the coordinates was performed.

For the massless case Eqs. (16) and (18) decouple and can be solved. Similarly, as was done in [20] for the massless case it is possible to pull a factor $1 / \rho_{+}$out of Eqs. (16) and (18). Let us note that Eqs. (17) and (19) do not explicitly depend on the variable $r$. It means that the variables $r$ and $\theta$ can be separated in the vicinity of the horizon. So the function $W(r, \theta)$ is represented in the form
$W(r, \theta)=W(r)+\Theta(\theta)$.

When $A=0$, Eq. (16) leads to

$W_{r}(r, \theta)=\frac{R_{+}^{2} E-\Xi a J-q\left(Q_{\mathrm{e}} r_{+} \mp Q_{\mathrm{m}} a\right)}{\Delta_{r}\left(r_{+}\right)\left(r-r_{+}\right)}$.

Having integrated around the pole, and taking the imaginary part of the action, we obtain

$\operatorname{Im} W_{+}=\frac{\pi R_{+}^{2}\left(E-\Xi \Omega_{+} J-\frac{q}{R_{+}^{2}}\left(Q_{\mathrm{e}} r_{+} \mp Q_{\mathrm{m}} a\right)\right)}{\Delta_{r}\left(r_{+}\right)}$,

where $\Omega_{+}=a / R_{+}^{2}$ is the angular velocity at the horizon.

Similarly, when $B=0$ we write

$W_{r}(r, \theta)=-\frac{R_{+}^{2} E-\Xi a J-q\left(Q_{\mathrm{e}} r_{+} \mp Q_{\mathrm{m}} a\right)}{\Delta_{r}\left(r_{+}\right)\left(r-r_{+}\right)}$.

And after integration the result is as follows:

$\operatorname{Im} W_{-}=-\frac{\pi R_{+}^{2}\left(E-\Xi \Omega_{+} J-\frac{q}{R_{+}^{2}}\left(Q_{\mathrm{e}} r_{+} \mp Q_{\mathrm{m}} a\right)\right)}{\Delta_{r}\left(r_{+}\right)}$.

As was argued in Refs. [7-9,20], the probabilities of crossing the horizon are defined by the imaginary part of the action:

$P_{\text {out }} \propto \exp \left[-2 \operatorname{Im} W_{+}\right], \quad P_{\text {in }} \propto \exp \left[-2 \operatorname{Im} W_{-}\right]$.

The resulting tunneling probability is represented as the ratio of the probabilities (25):

$\Gamma \propto \frac{P_{\text {out }}}{P_{\text {in }}}=\frac{\exp \left[-2 \operatorname{Im} W_{+}\right]}{\exp \left[-2 \operatorname{Im} W_{-}\right]}=\exp \left[-4 \operatorname{Im} W_{+}\right]$.

Substituting relation (22) into (26) we obtain

$\Gamma=\exp \left(-4 \pi \frac{R_{+}^{2}\left(E-\Xi \Omega_{+} J-\frac{q}{R_{+}^{2}}\left(Q_{\mathrm{e}} r_{+} \mp Q_{\mathrm{m}} a\right)\right)}{\Delta_{r}\left(r_{+}\right)}\right)$.

It should be noted that the obtained probabilities do not break unitarity. This is due to the fact that the temporal part of the action also gives a contribution to the emission and absorption probabilities [29-31], and the correct value of $\Gamma$ is obtained. 
As a result the temperature takes the form

$T=\frac{\Delta_{r}\left(r_{+}\right)}{4 \pi R_{+}^{2}}=\frac{\left(r_{+}\left(1-a^{2} / l^{2}\right)-2 r_{+}^{3} / l^{2}-M\right)}{2 \pi\left(r_{+}^{2}+a^{2}\right)}$.

In the massive case $(m \neq 0)$, the system of equations (16) and (18) cannot be decoupled and tunneling should be considered more carefully. First, we use these two equations to find an equation relating the parameters $A$ and $B$ in terms of known quantities. Having subtracted $B \times(18)$ from $A \times(16)$ we arrive at

$$
\begin{aligned}
& \frac{2 A B}{\rho_{+}}\left[\frac{R_{+}^{2} E-\Xi a J-q\left(Q_{\mathrm{e}} r_{+} \mp Q_{\mathrm{m}} a\right)}{\sqrt{\Delta_{r}\left(r_{+}\right)\left(r-r_{+}\right)}}\right] \\
& +m\left(A^{2}-B^{2}\right)=0
\end{aligned}
$$

and as a result trajectories. In spite of that qualitative difference, calculations of the Hawking temperature at the cosmological horizon can be made in the same way as at the event horizon. To obtain the expression for the Hawking temperature at the cosmological horizon one should replace the radius of the event horizon in formula (28) by the cosmological one $\left(r_{+} \rightarrow r_{\mathrm{c}}\right)$. So we have

$T=\frac{\Delta_{r}\left(r_{\mathrm{c}}\right)}{4 \pi R_{\mathrm{c}}^{2}}=\frac{\left(r_{\mathrm{c}}\left(1-a^{2} / l^{2}\right)-2 r_{\mathrm{c}}^{3} / l^{2}-M\right)}{2 \pi\left(r_{\mathrm{c}}^{2}+a^{2}\right)}$.

The relations for the temperature at the event horizon (28) and at the cosmological one take the same form as in the case of the black hole with only an electric charge [19]. It should be noted that the radii of the horizons depend on both the electric and the magnetic charge, so our formulas are consistent with the relations given in Ref. [19] when $Q_{\mathrm{m}} \rightarrow 0$.

$$
\frac{A}{B}=\frac{-\left(R_{+}^{2} E-\Xi a J-q\left(Q_{\mathrm{e}} r_{+} \mp Q_{\mathrm{m}} a\right)\right) \pm \sqrt{\left(R_{+}^{2} E-\Xi a J-q\left(Q_{\mathrm{e}} r_{+} \mp Q_{\mathrm{m}} a\right)\right)^{2}+m^{2} \rho_{+}^{2} \Delta_{r}\left(r_{+}\right)\left(r-r_{+}\right)}}{m \rho_{+} \sqrt{\Delta_{r}\left(r_{+}\right)\left(r-r_{+}\right)}} .
$$

When $r \rightarrow r_{+}$the ratio $A / B$ can tend to 0 or $-\infty$ (see also [20]). When $A / B \rightarrow 0$ then $A \rightarrow 0$ the Eq. (18) is solved in terms of $m$ and the result is inserted into (16). It should be noted that the resulting expression does not depend on the variable $\theta$. So we obtain

$$
\begin{aligned}
& W_{r}(r, \theta)=W_{+}^{\prime}(r) \\
& =\frac{R_{+}^{2}\left(E-\Xi \Omega_{+} J-\frac{q}{R_{+}^{2}}\left(Q_{\mathrm{e}} r_{+} \mp Q_{\mathrm{m}} a\right)\right)}{\Delta_{r}\left(r_{+}\right)\left(r-r_{+}\right)} \frac{1+\frac{A^{2}}{B^{2}}}{1-\frac{A^{2}}{B^{2}}}
\end{aligned}
$$

The result that is found after integration around the pole is the same as in the massless case, since $A / B \rightarrow 0$ at the horizon. When $B \rightarrow 0$ the relation (31) can be rewritten as follows:

$$
\begin{aligned}
& W_{r}(r, \theta)=W_{-}^{\prime}(r) \\
& =-\frac{R_{+}^{2}\left(E-\Xi \Omega_{+} J-\frac{q}{R_{+}^{2}}\left(Q_{\mathrm{e}} r_{+} \mp Q_{\mathrm{m}} a\right)\right)}{\Delta_{r}\left(r_{+}\right)\left(r-r_{+}\right)} \frac{1+\frac{B^{2}}{A^{2}}}{1-\frac{B^{2}}{A^{2}}}
\end{aligned}
$$

Similarly the result after integration gets no correction in addition to the massless case. As a consequence the temperature for the emitted massive fermions is the same as for the massless fermions, and it given by the relation (28).

It is known that Hawking radiation in de Sitter space can also appear at the cosmological horizon. In contrast to the emission of particles at the event horizon, Hawking radiation at the cosmological horizon is caused by incoming particles whereas outgoing particles move along classically permitted

\section{Action for the emitted particles}

Equations (16)-(19) allow one to obtain an explicit expression for the action of the emitted particles. As was already shown, angle and radial variables are separated near the horizon. So the radial and angular parts of the action can be obtained independently. Having used the relation (20), Eq. (16) can be rewritten in the form

$$
\begin{aligned}
W^{\prime}(r)= & \frac{R_{+}^{2} E-\Xi a J-q\left(Q_{\mathrm{e}} r_{+} \mp Q_{\mathrm{m}} a\right)}{\Delta_{r}\left(r_{+}\right)\left(r-r_{+}\right)} \\
& +\frac{A m \rho_{+}}{B \sqrt{\Delta_{r}\left(r_{+}\right)\left(r-r_{+}\right)}} .
\end{aligned}
$$

After integration we obtain

$$
\begin{aligned}
W_{+}(r)= & \frac{R_{+}^{2} E-\Xi a J-q\left(Q_{\mathrm{e}} r_{+} \mp Q_{\mathrm{m}} a\right)}{\Delta_{r}\left(r_{+}\right)} \ln \left(r-r_{+}\right) \\
& +\int \frac{A m \rho_{+}}{B \sqrt{\Delta_{r}\left(r_{+}\right)\left(r-r_{+}\right)}} \mathrm{d} r .
\end{aligned}
$$

In order to find relation for radial part of the action for incoming particles one should integrate Eq. (18). After integration we arrive at the relation

$$
\begin{aligned}
W_{-}(r)= & -\frac{R_{+}^{2} E-\Xi a J-q\left(Q_{\mathrm{e}} r_{+} \mp Q_{\mathrm{m}} a\right)}{\Delta_{r}\left(r_{+}\right)} \ln \left(r-r_{+}\right) \\
& +\int \frac{B m \rho_{+}}{A \sqrt{\Delta_{r}\left(r_{+}\right)\left(r-r_{+}\right)}} \mathrm{d} r
\end{aligned}
$$


For the angular part, relation (17) can be represented in the form

$$
\begin{aligned}
\Theta^{\prime}(\theta)= & -i \frac{\left(\Xi J \mp q Q_{\mathrm{m}}\right)}{\sin \theta \Delta_{\theta}}+i \frac{\left(\Xi J \mp q Q_{\mathrm{m}}\right) a^{2} \sin \theta}{R_{+}^{2} \Delta_{\theta}} \\
& -i \frac{q Q_{\mathrm{e}} r_{+} a \sin \theta}{R_{+}^{2} \Delta_{\theta}}-i \frac{q Q_{\mathrm{m}} \cos \theta}{\sin \theta \Delta_{\theta}} .
\end{aligned}
$$

Having integrated the last equation we obtain

$$
\begin{aligned}
\Theta(\theta)= & i \frac{\left(\Xi J \mp q Q_{\mathrm{m}}\right)}{1+\frac{a^{2}}{l^{2}}} \\
\times & \left(\frac{a}{l} \arctan \left(\frac{a \cos \theta}{l}\right)-\frac{1}{2} \ln \left|\frac{1+\cos \theta}{1-\cos \theta}\right|\right) \\
& -i \frac{a\left(\Xi J a-q\left(Q_{\mathrm{e}} r_{+} \mp Q_{\mathrm{m}} a\right)\right)}{R_{+}^{2}} \\
& \times \frac{l}{a} \arctan \left(\frac{a \cos \theta}{l}\right)+i \frac{q Q_{\mathrm{m}}}{2\left(1+\frac{a^{2}}{l^{2}}\right)} \\
& \times\left(\ln \left|1+\frac{a^{2}}{l^{2}} \cos ^{2} \theta\right|-\ln \left|1-\cos ^{2} \theta\right|\right) .
\end{aligned}
$$

Using the relations (36) and (38), one can write the action for outgoing massive particles. Similarly Eqs. (36) and (38) allow one to get the action for the incoming particles.

\section{Conclusions}

In this paper, we considered charged fermion tunneling from the electrically and magnetically charged Kerr-Newman-de Sitter black hole. Using the Kerner-Mann approach [20], we successfully recovered the black hole's temperature. It was shown that similarly to the case when the black hole carries only an electric charge, inclusion of an additional magnetic charge does not spoil the separability of the Dirac equation in the vicinity of the horizons. So the relations for the temperature are obtained in the same manner and take almost the same form as in the case of an electrically charged black hole. We also note that for the temperature an explicit dependence on the magnetic charge is hidden in the definition of the radii of the horizons.

We also obtained relations for the radial and angular parts of the action. Those relations might be helpful if one tries to find corrections to the spectrum of emitted particles. Here we have an explicit dependence on the electric as well as magnetic charges, so these terms might have a different influence on the spectrum of the emitted particles.

Another issue that still remains open is taking into account higher orders of the WKB corrections. This problem is connected with the calculation of terms caused by the spin connection. These terms might affect the separability and tractability of the Dirac equation and this problem requires additionally careful consideration.

Open Access This article is distributed under the terms of the Creative Commons Attribution License which permits any use, distribution, and reproduction in any medium, provided the original author(s) and the source are credited.

Article funded by SCOAP ${ }^{3}$ and licensed under CC BY 4.0

\section{References}

1. S.W. Hawking, Commun. Math. Phys. 43, 199 (1975)

2. R. Brout, S. Massar, R. Parentani, P. Spindel, Phys. Rep. 260, 329 (1995)

3. P. Kraus, F. Wilczek, Nucl. Phys. B 433, 403 (1995)

4. P. Kraus, F. Wilczek, Nucl. Phys. B 437, 231 (1995)

5. M.K. Parikh, F. Wilczek, Phys. Rev. Lett. 85, 5042 (2000)

6. M. Angheben, M. Nadalini, L. Vanzo, S. Zerbini, JHEP 0505, 014 (2005)

7. K. Srinivasan, T. Padmanabhan, Phys. Rev. D 66, 024007 (2002)

8. S. Shankaranarayananan, K. Srinivasan, T. Padmanabhan, Mod. Phys. Lett. A 16, 571 (2001)

9. S. Shankaranarayananan, K. Srinivasan, T. Padmanabhan, Class. Quant. Grav. 19, 2671 (2002)

10. Q.-Q. Jiang, S.-Q. Wu, X. Cai, Phys. Rev. D. 73, 064003 (2006)

11. J. Zhang, Z. Zhao, Phys. Lett. B 638, 110 (2006)

12. R. Kerner, R. Mann, Phys. Rev. D 73, 104010 (2006)

13. R. Kerner, R. Mann, Phys. Rev. D 75, 084022 (2007)

14. S.-Q. Wu, Q.-Q. Jiang, JHEP 0603, 079 (2006)

15. R. di Criscienzo, M. Nadalini, L. Vanzo, S. Zerbini, G. Zoccatelli, Phys. Lett. B 657, 107 (2007)

16. L. Vanzo, G. Aquaviva, R. Di Crisienzo Class. Quant. Grav. 28, 183001 (2011)

17. R. Kerner, R.B. Mann, Class. Quant. Grav. 25, 095014 (2008)

18. X.-X. Zeng, S.-Z. Yang, Gen. Rel. Grav. 40, 2107 (2008)

19. D.-Y. Chen, Q.-Q. Jiang, X.-T. Zu, Phys. Lett. B 665, 106 (2008)

20. R. Kerner, R.B. Mann, Phys. Lett. B 665, 277 (2008)

21. D.-Y. Chen, Q.-Q. Jiang, X.-T. Zu, Class. Quant. Grav. 25, 205022 (2008)

22. R. Li, J.-R. Ren, Phys. Lett. B 661, 370 (2008)

23. D.-Y. Chen, H. Yang, X.-T. Zu, Phys. Lett. B 681, 463 (2009)

24. M. Liu, J. Lu, J. Lu, Class. Quant. Grav. 28, 125024 (2011)

25. M. Rehman, K. Saifullah, JCAP 1103, 001 (2011)

26. M. Sharif, W. Javed, Eur. Phys. J. C 72, 1997 (2012)

27. J. Ahmed, K. Saifullah, JCAP 11, 023 (2011)

28. I. Semiz, Phys. Rev. D 46, 5414 (1992)

29. E.T. Akhmedov, V. Akhmedova, D. Singleton, Phys. Lett. B 642, 124 (2006)

30. V.A. Akhmedova, T. Pilling, D. Singleton, Phys. Lett. B 666, 269 (2008)

31. E.T. Akhmedov, V. Akhmedova, D. Singleton, Int. J. Mod. Phys. D 17, 2453 (2008) 